\title{
is
}

\section{The Role of the Primary Information on Importance of the Last Information in Decision Making}

\author{
Maryam Tohidi-Moghaddam ${ }^{1,2}$, Sajjad Zabbah², Reza Ebrahimpour ${ }^{1^{*}}$ \\ ${ }^{1}$ Faculty of Computer Engineering, Shahid Rajaee Teacher Training University, Tehran, Iran \\ ${ }^{2}$ School of Cognitive Science, Institute for Research in Fundamental Science (IPM), Tehran, Iran
}

\section{ABSTRACT}

Introduction: Most decisions are based on the accumulation of discrete pieces of evidence.

This evidence has usually been separated with the various intervals. Indeed, how the brain gathers and combines distinct pieces of information received at different times is need to be clarified. In order to investigate the kinship between brain function and human behavior, the behavioral experimental studies could be designed. Previous studies demonstrated that subjects gather and effectively combine discrete evidence to improve their accuracy. In addition, it has been shown that the latest information has a larger influence on decisions. However, it remains unclear that why this larger influence of the later pulses occurs and what can affect this influence. Materials and Methods: Dealing with these questions a perceptual decision-making task has been implemented by the psychophysics' toolbox in MATLAB. Subjects, during the task, were instructed to report the direction of motion in a noisy random dot stimulus with certain keys. Stimuli were presented in continuous (one pulse) or discrete (two continuousness pulses separated with different intervals) form. Each of these two forms of stimuli was presented randomly during each session. Each session has been included 300 trials. Each subject has done 3600 trials. Data have been analyzed by regression models. Results: We observed that in double-pulse trials, the strength of the second pulse was more crucial in the accuracy of responses compared to the first pulse. In addition, this accuracy was dependent on the differences between the strength of the first and the last pulses. Conclusion: These findings suggest that a key factor which affects the importance of pulses is the strength of the previous pulse. As the difference between the motion strength increases, the effect of the second pulse on choice accuracy enhanced.

\section{Key words:}

1. Psychophysics

2. Neurosciences

3. Decision Making

*Corresponding Author: Reza Ebrahimpour

E-mail: rebrahimpour@srttu.edu

doi: 10.18869/acadpub.shefa.4.4.26 


\title{
نقش اطلاعات اوليه در اهميت اطلاعات نهايى در تصميمزيرى
}

\author{
مريم توحيدى مقدّم ז'، سجّاد ذبّاح'، رضا ابر اهيميور "* \\ 'دانشكده مهندسى كامييوتر، دانشَاه تربيت دبير شهيد رجايى، تهران، ايران

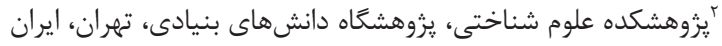

\section{Q}

مقدمه: بيشترين تصميمها مبنى بر جمع آورى بخشهاى گسستهاى از اطلاعات است. اين اطلاعات

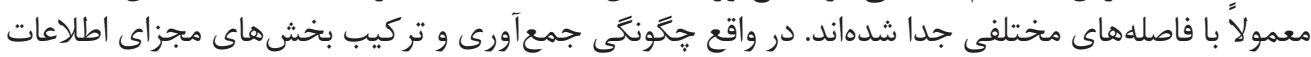

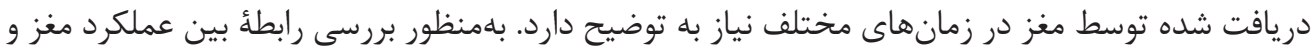

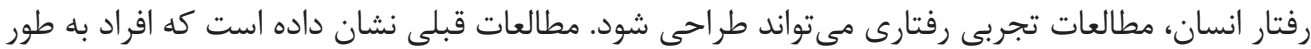

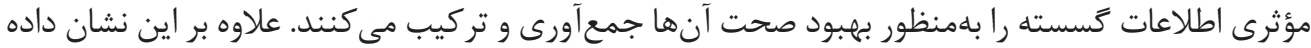

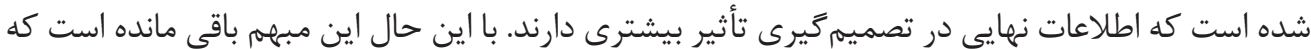

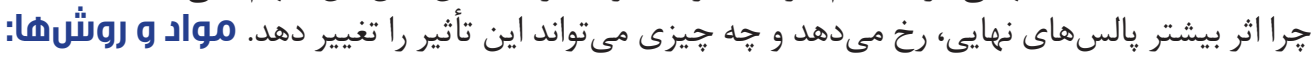

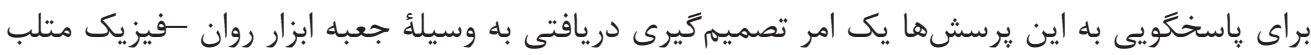

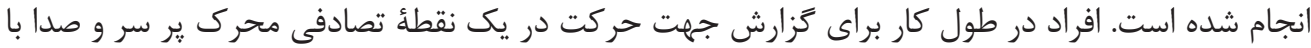

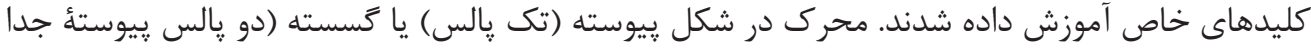

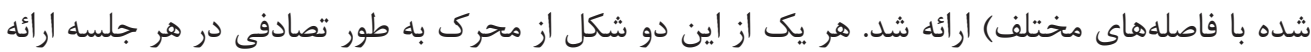

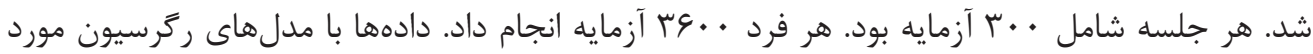

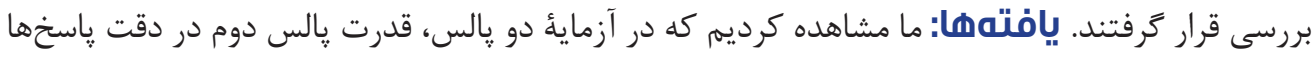

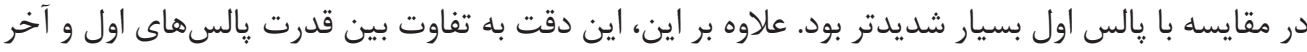

كليد وازهها:

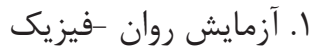

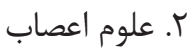

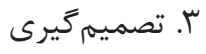

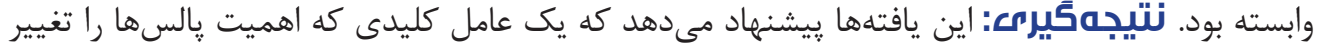

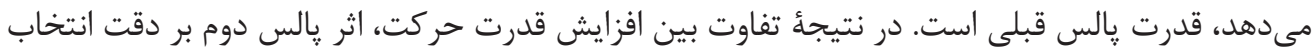

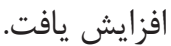




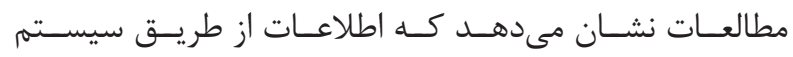

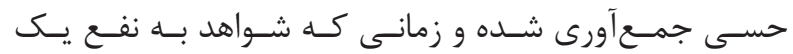

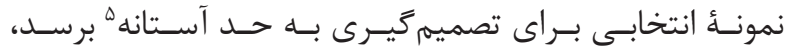

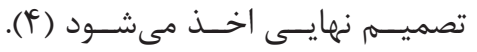

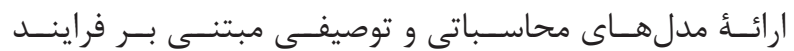

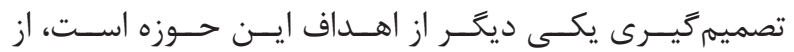

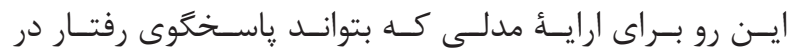

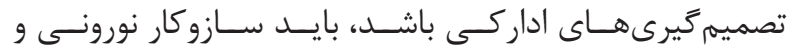

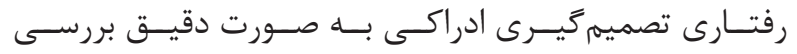

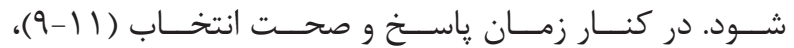

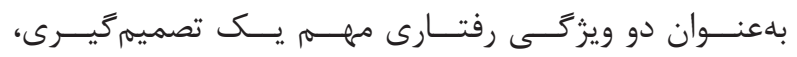

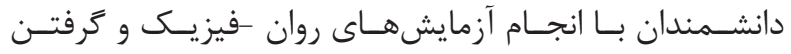

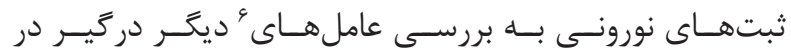

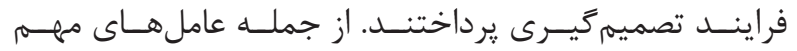

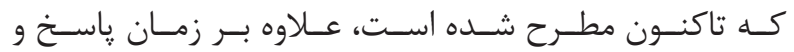

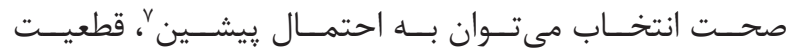

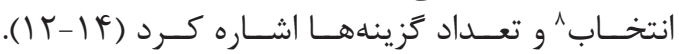

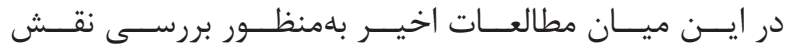

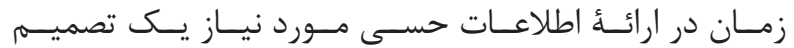

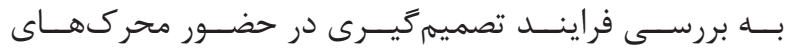

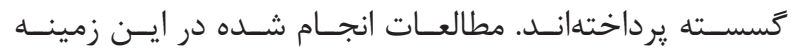

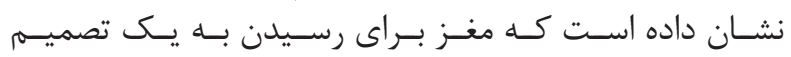

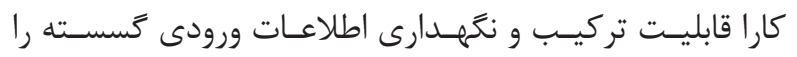

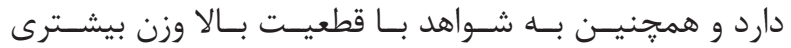

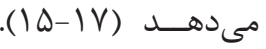

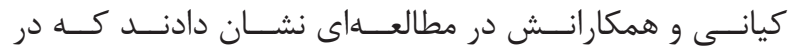

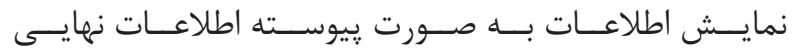

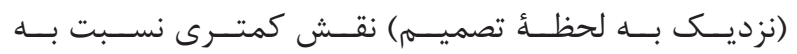

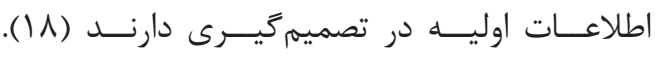

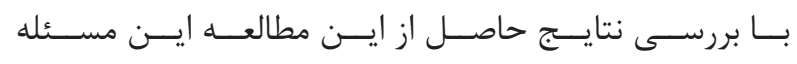

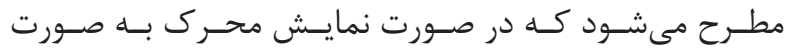

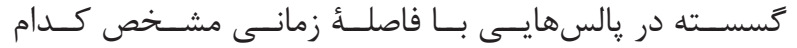

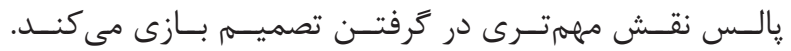

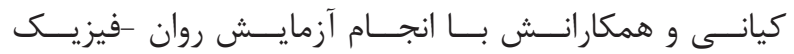

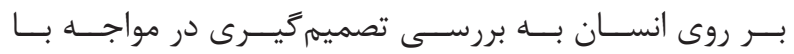

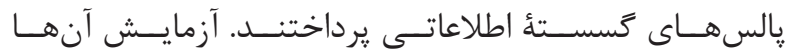

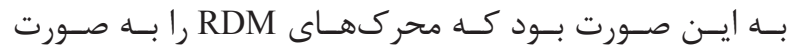

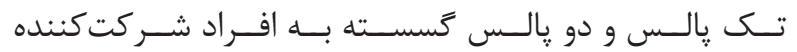

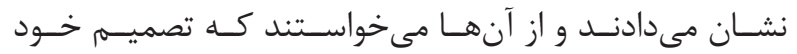

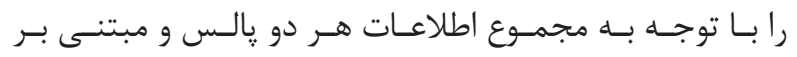

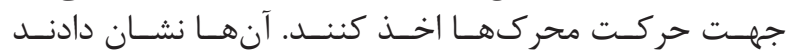

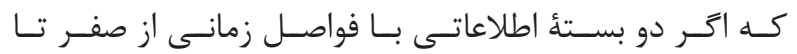

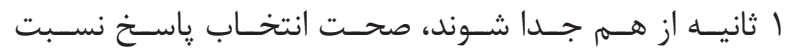

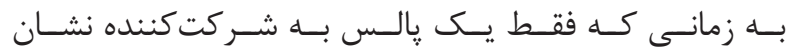

${ }^{1}$ Psychophysics

${ }^{2}$ Random dot motion

${ }^{3}$ Coherency

${ }^{4}$ Subject

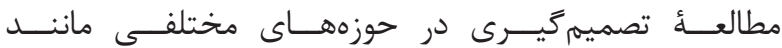

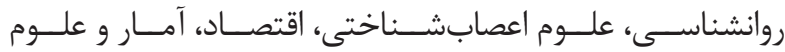

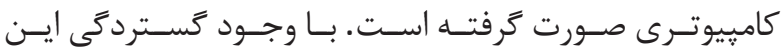

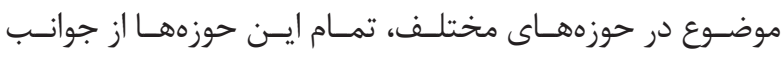

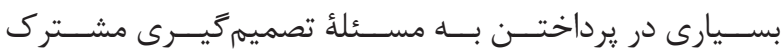

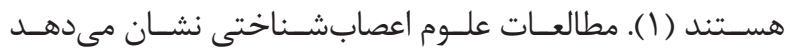

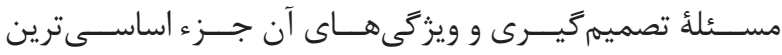

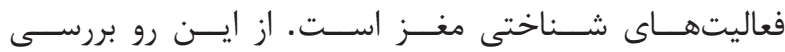

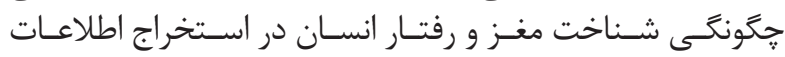

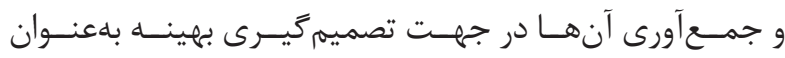

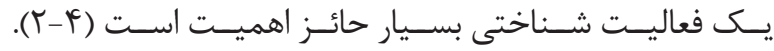

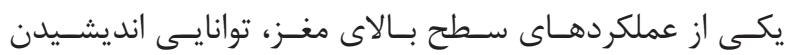

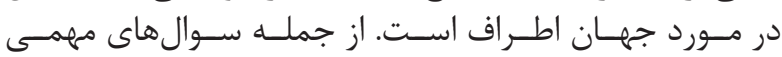

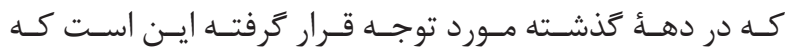

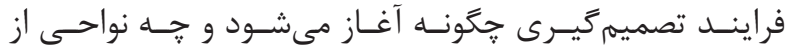

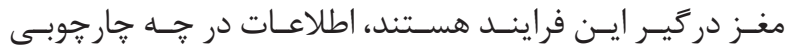

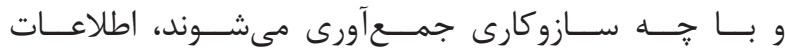

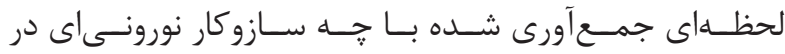

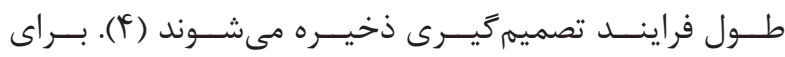

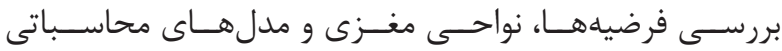

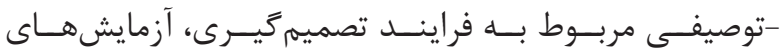

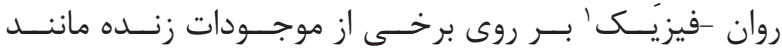

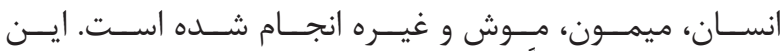

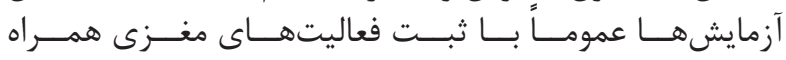

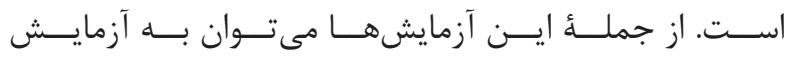

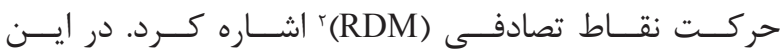

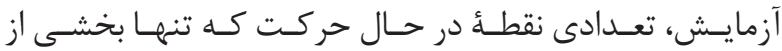

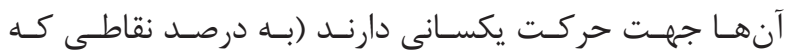

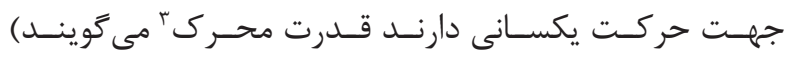

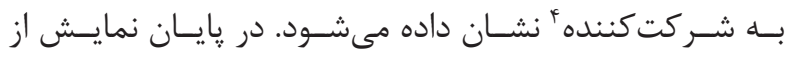

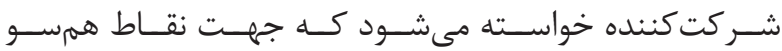

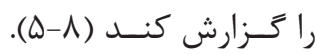

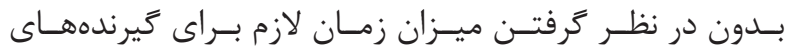

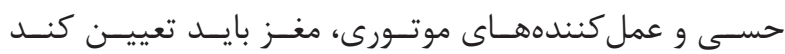

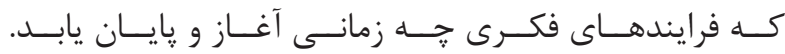

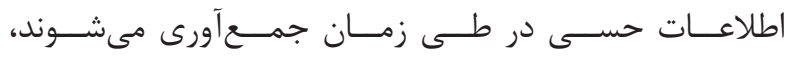

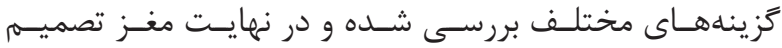

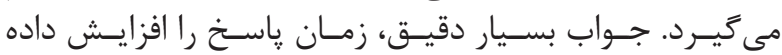

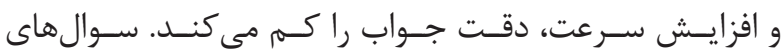

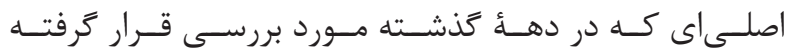

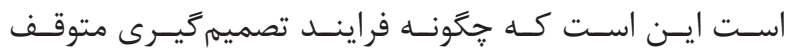

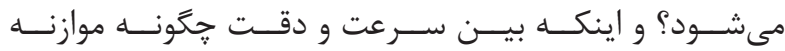

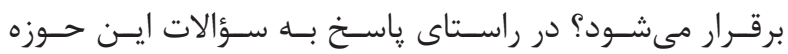

${ }^{5}$ Threshold

${ }^{6}$ Parameter

${ }^{7}$ Prior probability

${ }^{8}$ Choice certainty 


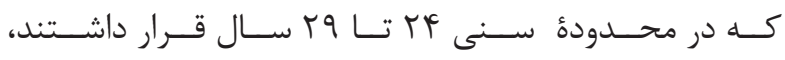

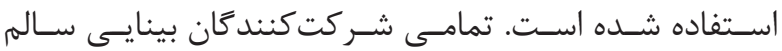

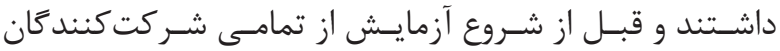

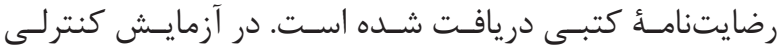

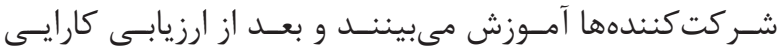

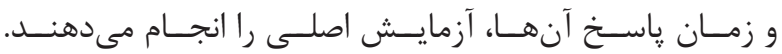

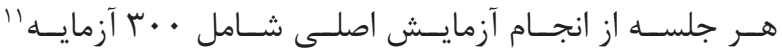

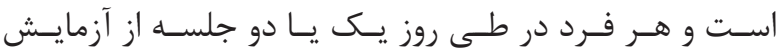

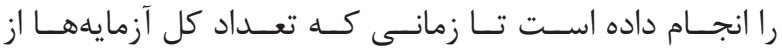

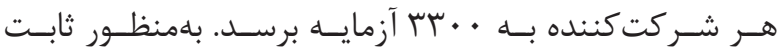

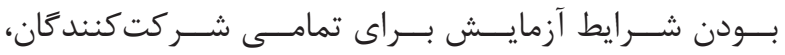

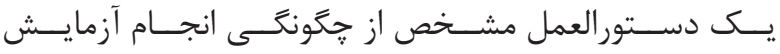

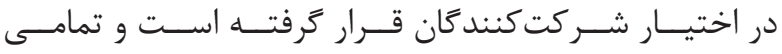

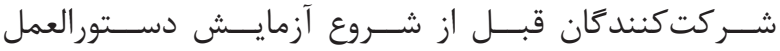

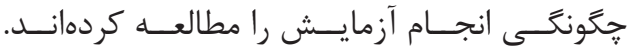

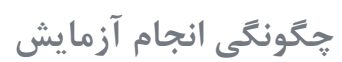

تمامسى شـركت كنند كان در يـك اتـاق نســـتاً تاريـك و عايـق

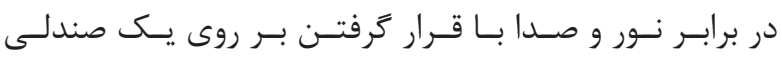

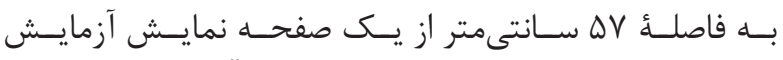

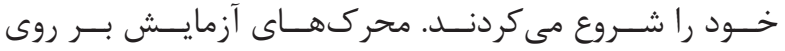

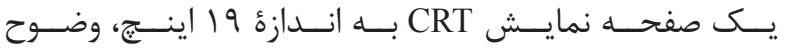

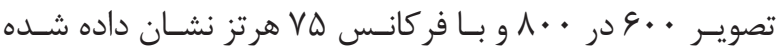

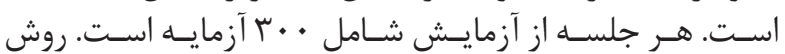

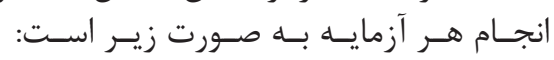

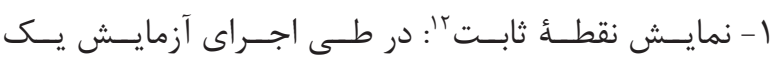

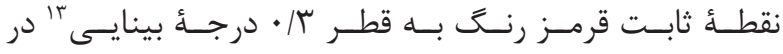

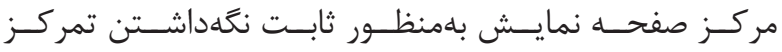

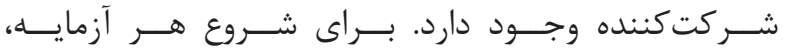

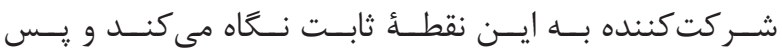

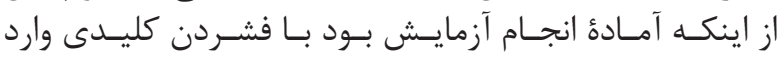

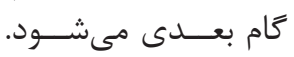

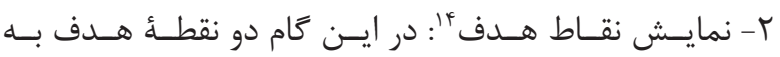

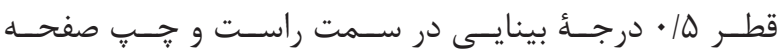

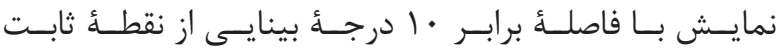

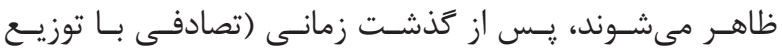

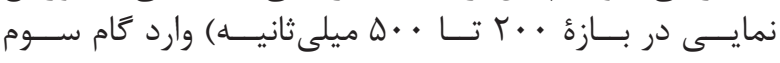

$$
\text { مى شـــود. }
$$

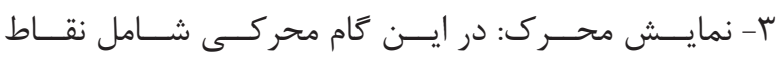

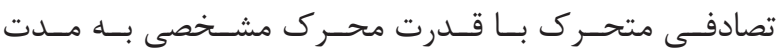

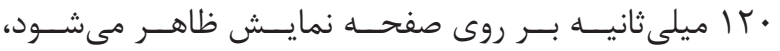

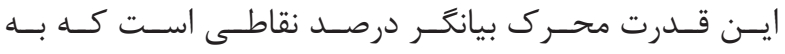

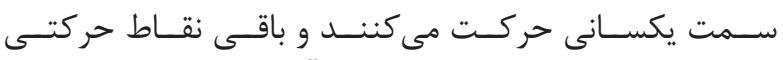

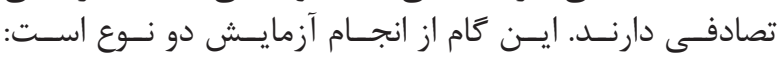

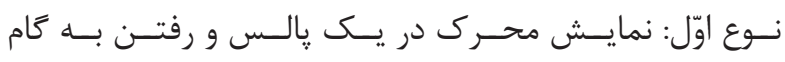

${ }^{9}$ Psychometric function

${ }^{10}$ Psychometric toolbox

${ }^{11}$ Trial

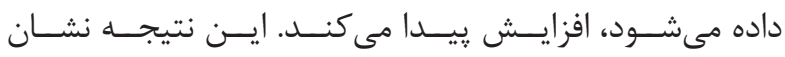

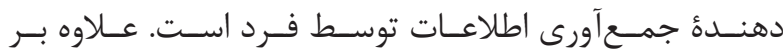

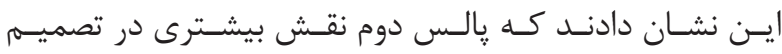

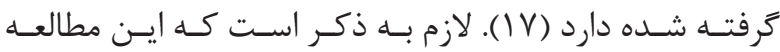

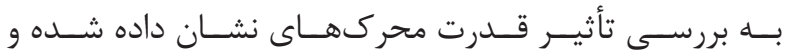

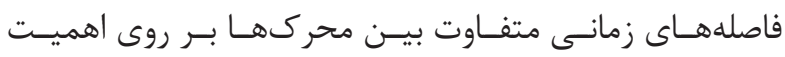

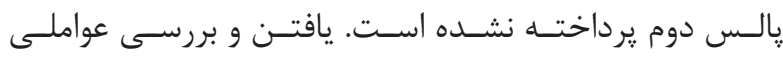

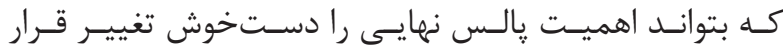

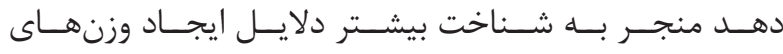

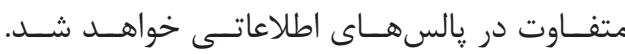

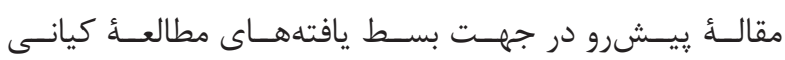

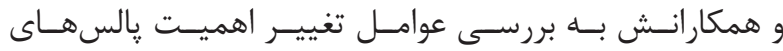

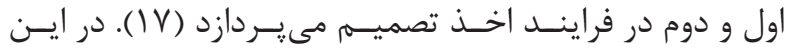

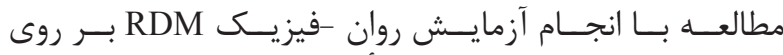

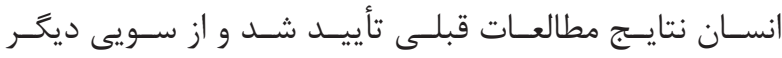

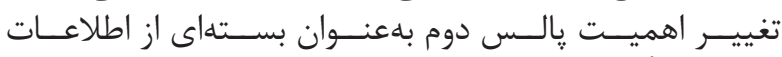

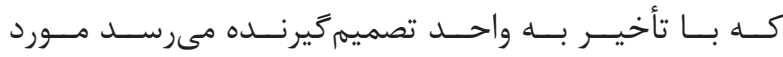

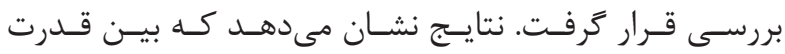

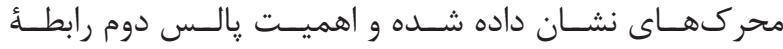

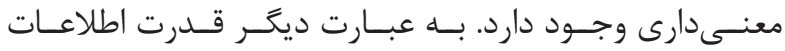

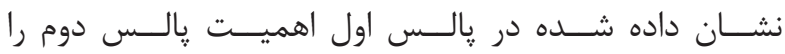

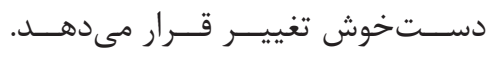

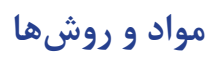
روش تحقيق

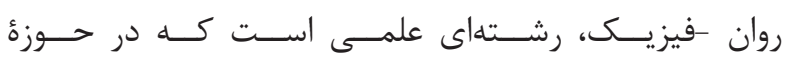

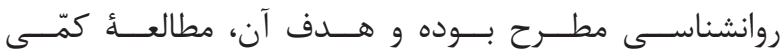

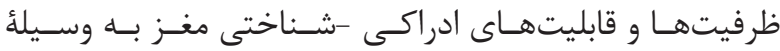

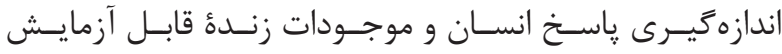

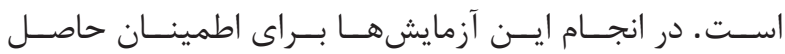

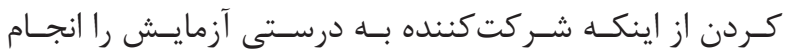

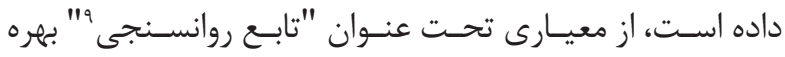

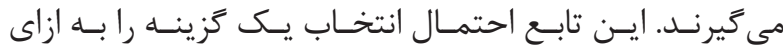

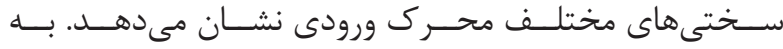

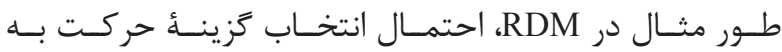

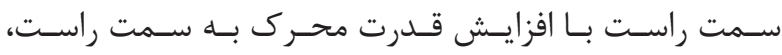
افزايــش مى سيابـــد.

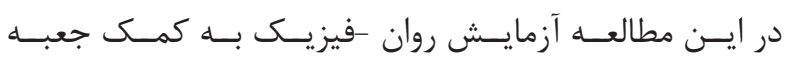

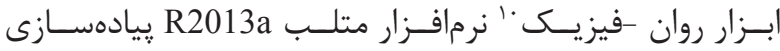

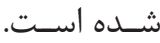
جمع آورى داده

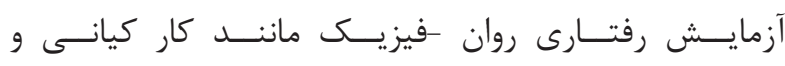

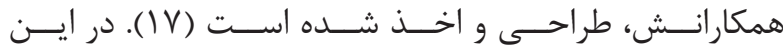

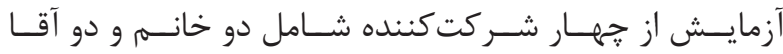

\footnotetext{
${ }^{12}$ Fixation poin

${ }^{13}$ Visual angle

${ }^{14}$ Target
} 


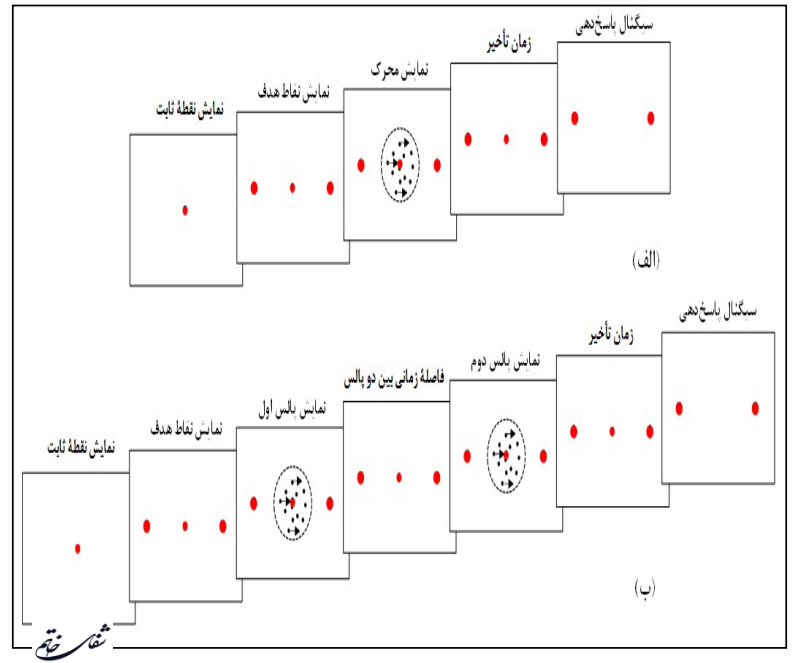

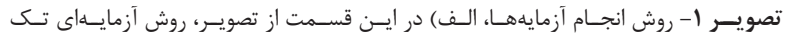

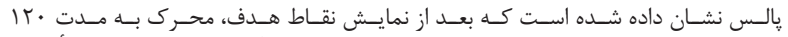

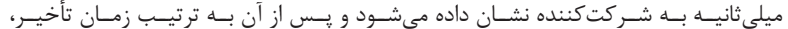

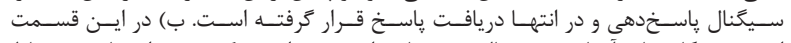

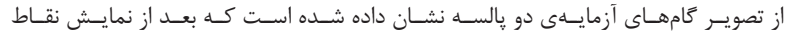

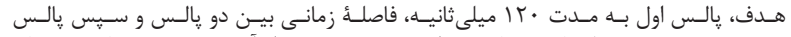

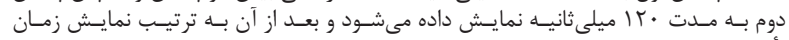

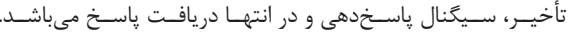

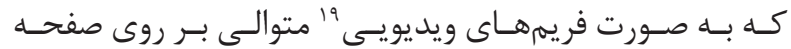

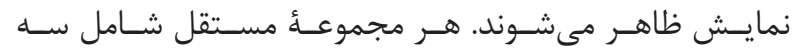

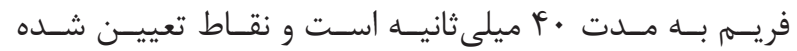

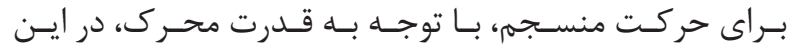

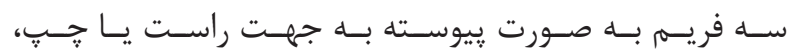

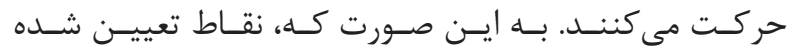

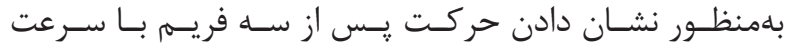

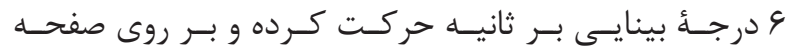

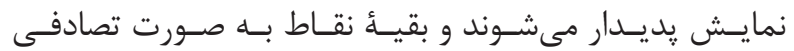

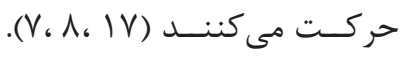
تحليل آمارى دادهها

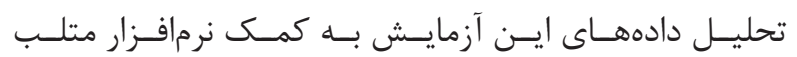

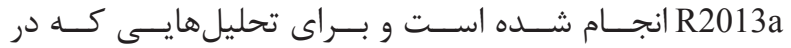

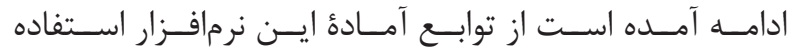

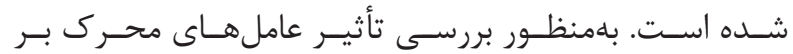

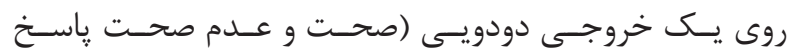

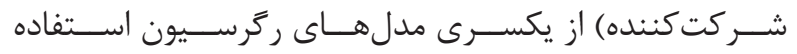

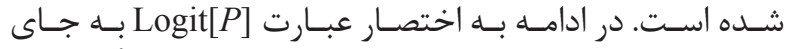

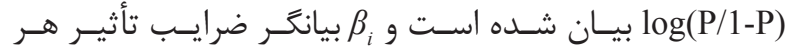

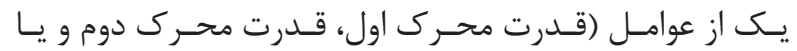
فاصلههــاى زمانـى ) هســتـند.

بــراى آزمايههـــاى تكى

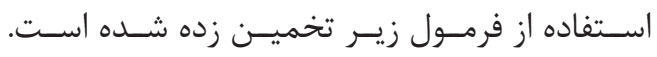

$\operatorname{Logit}\left[P_{\text {correct }}\right]=\beta_{0}+\beta_{1} C$

در ايـن معادلـه، C قـدرت محــــ اسـت و بهمنظــور بررسـى

${ }^{15}$ Delay

${ }^{16}$ Go signal

${ }^{17}$ Feedback

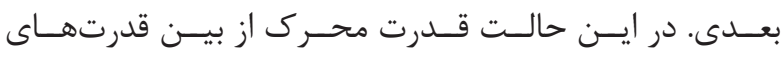

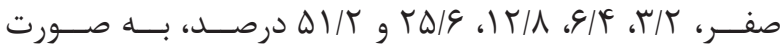

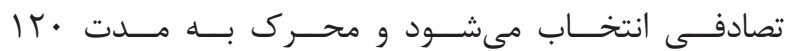

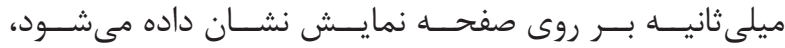

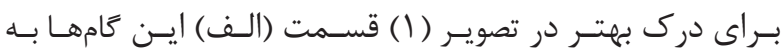

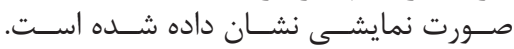

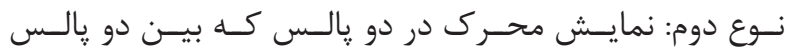

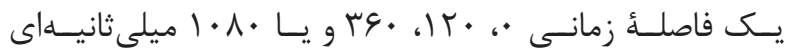

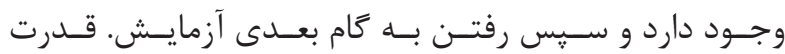

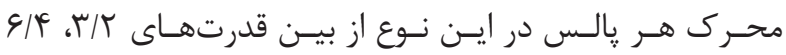

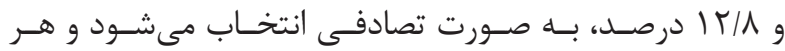

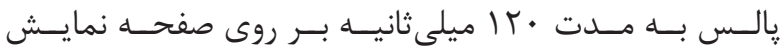

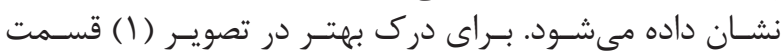

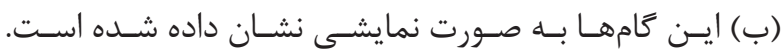

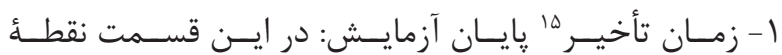

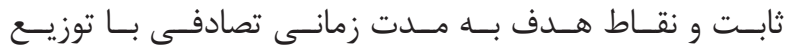

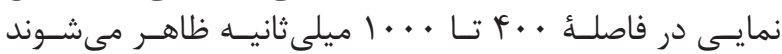

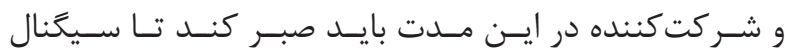

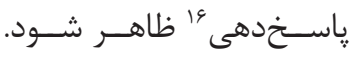

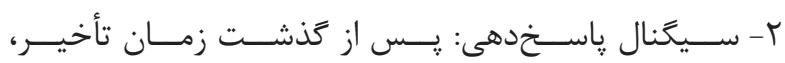

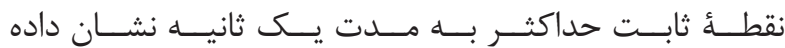

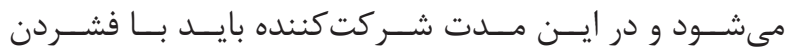

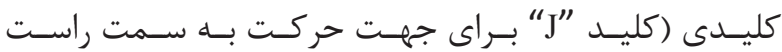

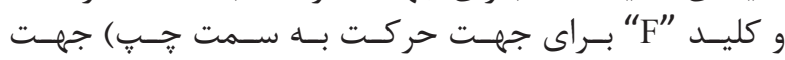

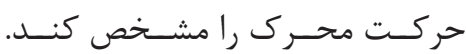

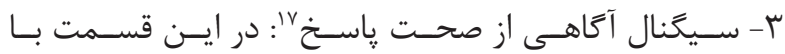

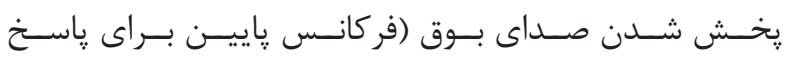

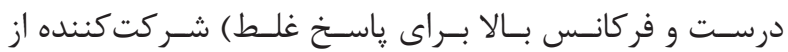

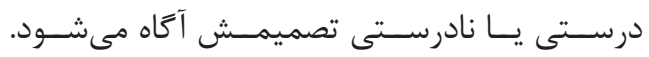

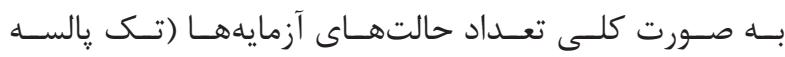

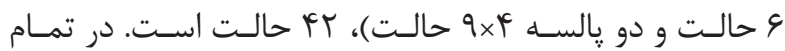

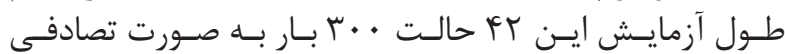

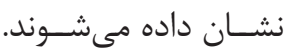
محرك بينايى

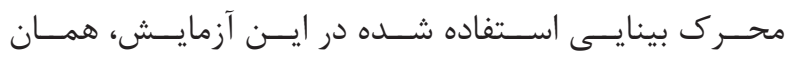

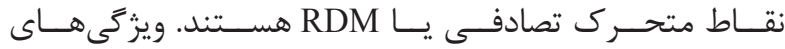

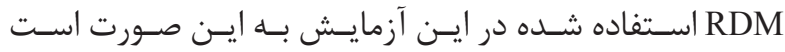

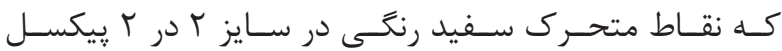

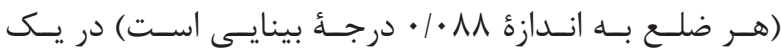

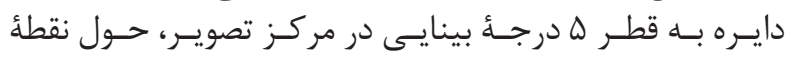

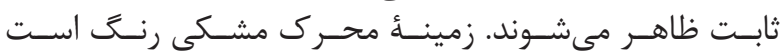

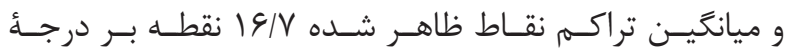

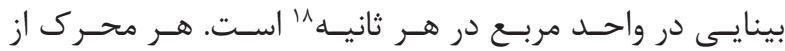

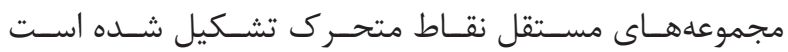

${ }^{18} \mathrm{Dots} /$ degree $2 / \mathrm{sec}$

${ }^{19}$ Video Frame

${ }^{20}$ Bias 


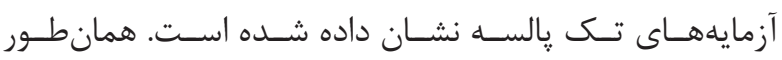

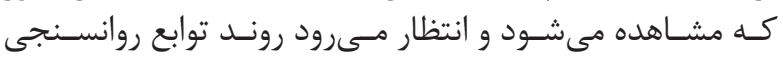

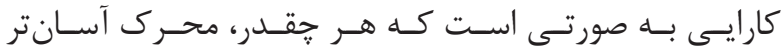

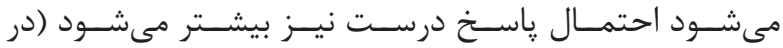

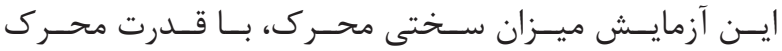

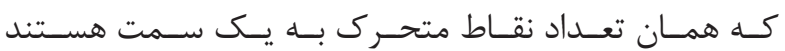

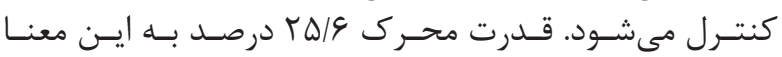

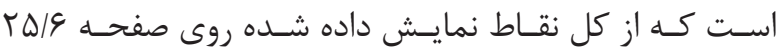

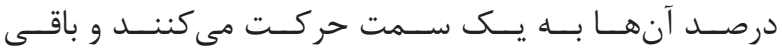

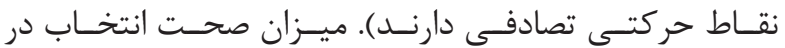

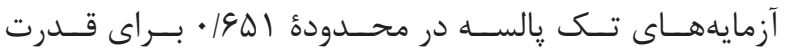

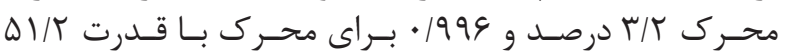

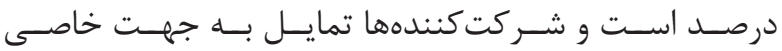

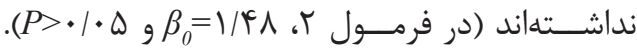

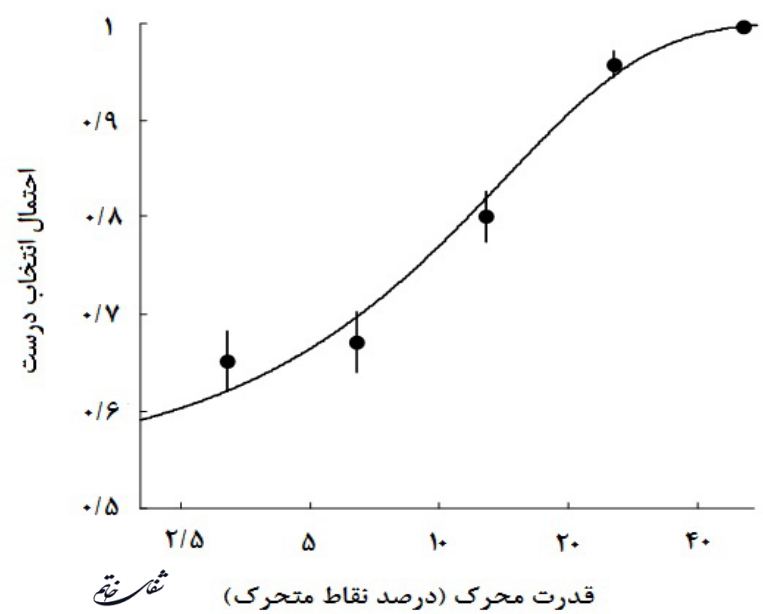

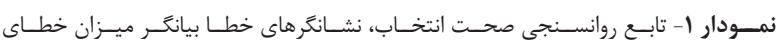

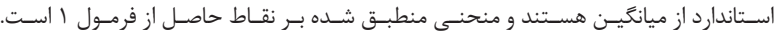

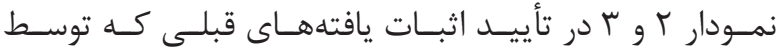

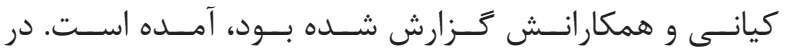

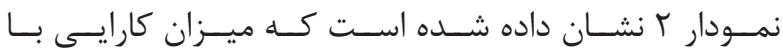

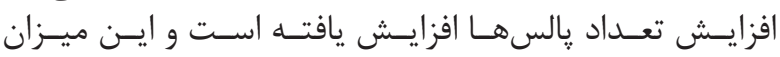

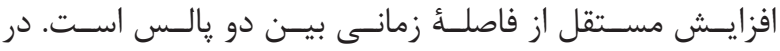

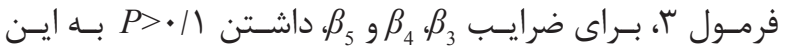

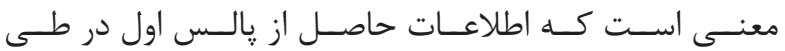

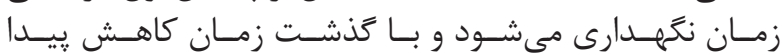

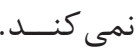

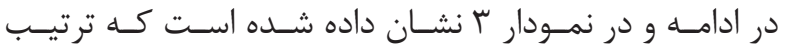

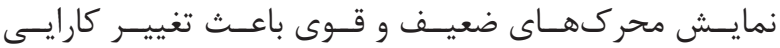

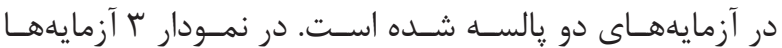

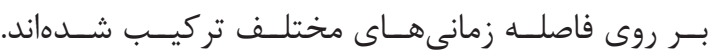

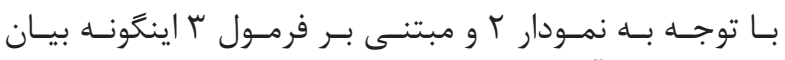

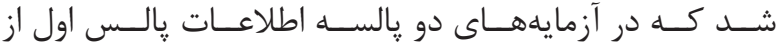

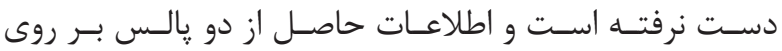

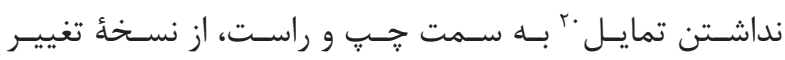

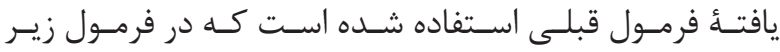
$\operatorname{Logit}\left[P_{\text {correct }}\right]=\beta_{\mathrm{o}}+\beta_{1} C_{ \pm}$

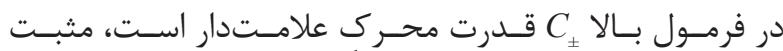

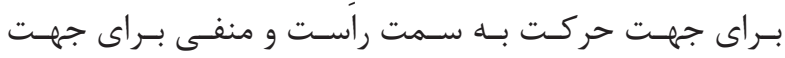

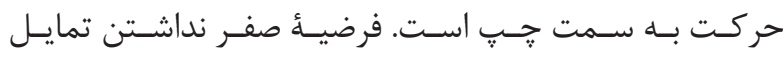

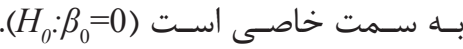

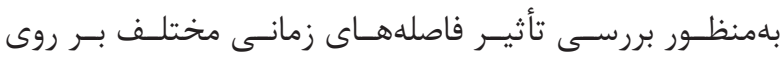

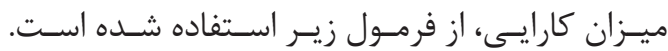

$\operatorname{Logit}\left[P_{\text {correct }}\right]=\beta_{0}+\beta_{1} C_{1}+\beta_{2} C_{2}+\beta_{3} T+\beta_{4} C_{1} T+\beta_{5} C_{2} T$

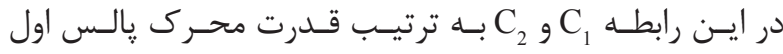

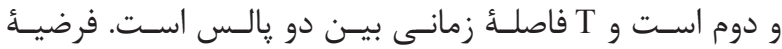

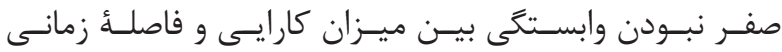
بيـن دو يالـس اسـت (بـ

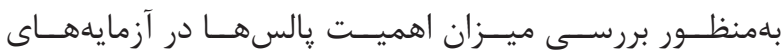

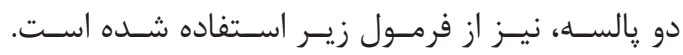
$\operatorname{Logit}\left[P_{\text {correct }}\right]=\beta_{0}+\beta_{1} C_{1}+\beta_{2} C_{2}$

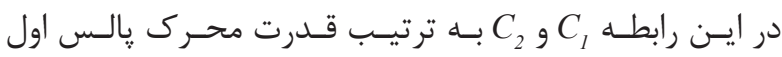

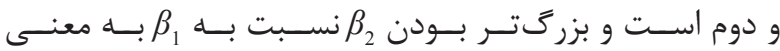

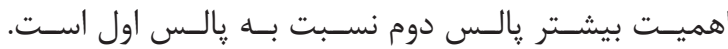

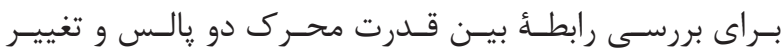

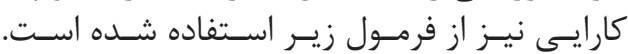

$\operatorname{Logit}\left[P_{\text {correct }}\right]=\beta_{0}+\beta_{1}\left[C_{1}+C_{2}\right]+\beta_{2} C_{2} / C_{1}$

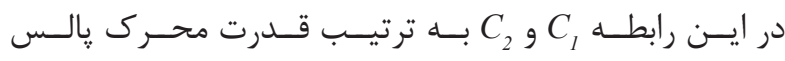

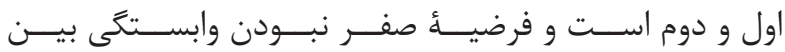

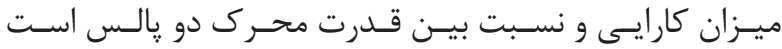
. $\left(\mathrm{H}_{0}: \beta_{2}=0\right)$

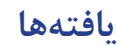

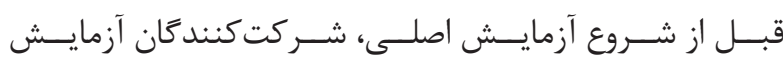

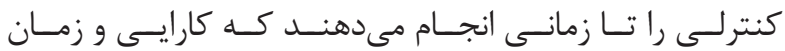

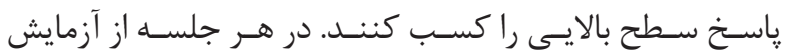

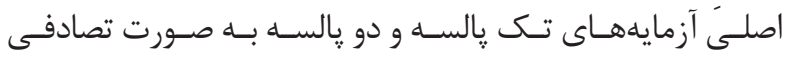

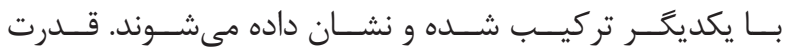

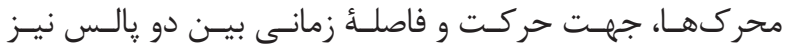

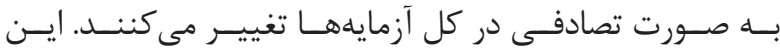

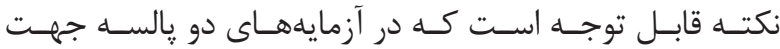

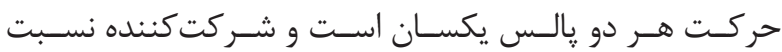
بـهـ ايـن مســــله آكاه اسـت. در نمـودار ا، تابـع روانسـنجى احتمــال انتخــاب درسـت در 


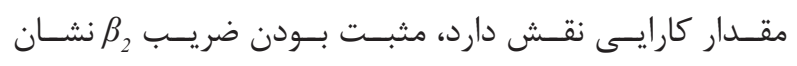

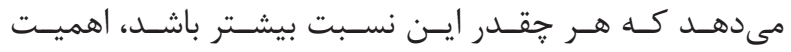

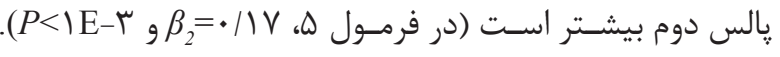

\section{بحث و نتيجه كَيرى}

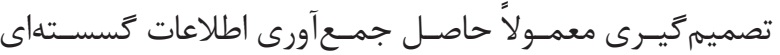

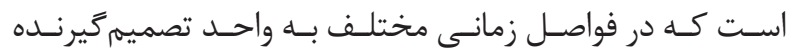

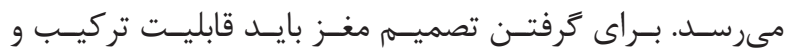

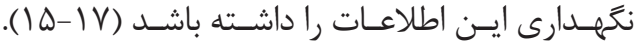

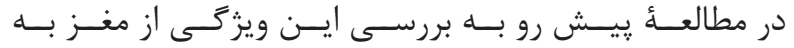

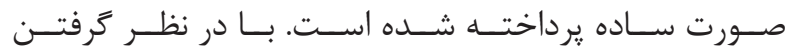

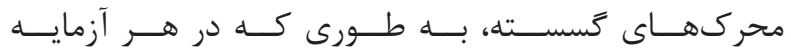

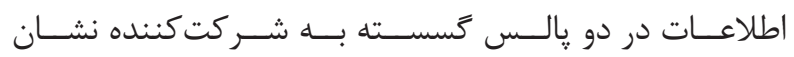

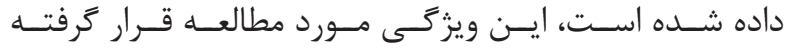

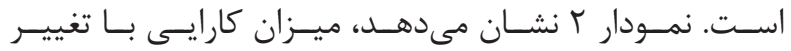

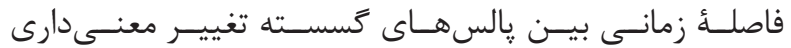

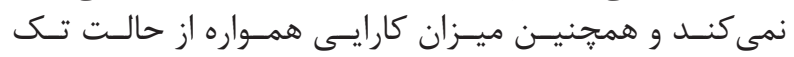

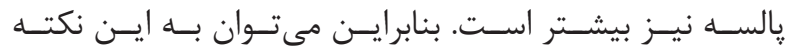

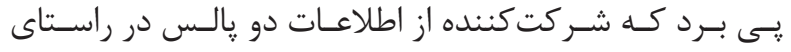

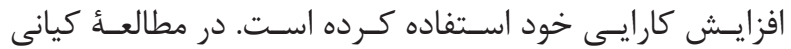

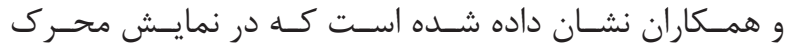

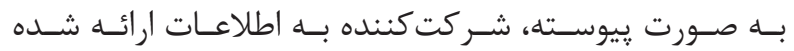

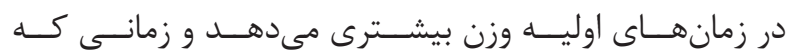

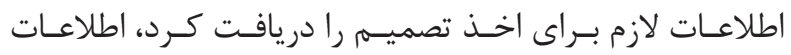

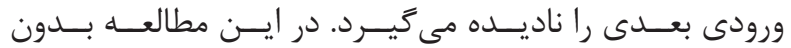

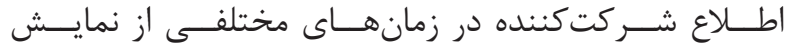

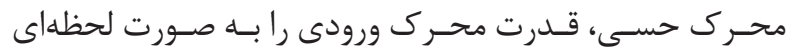

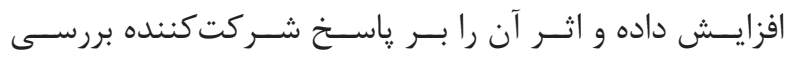

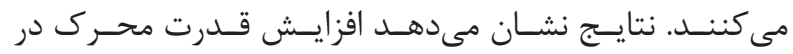

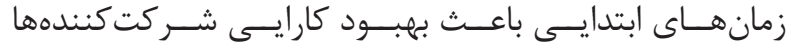

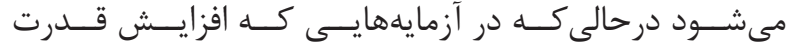

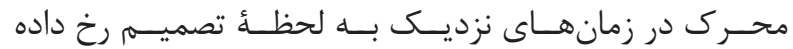

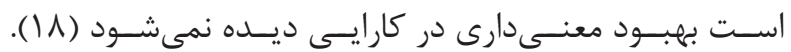

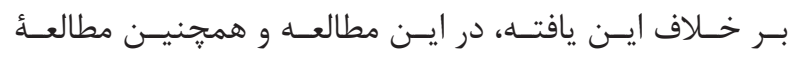

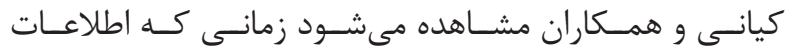

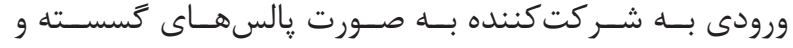

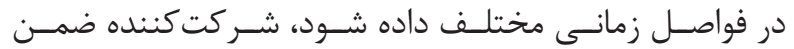

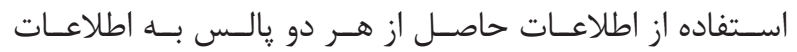

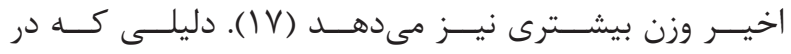

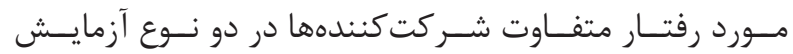

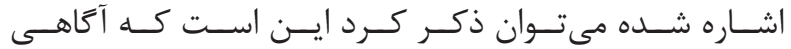

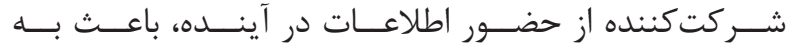

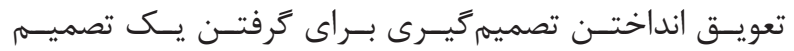

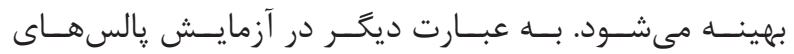

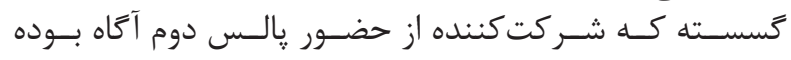

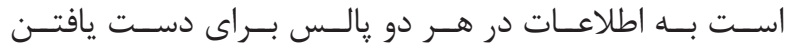

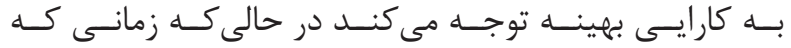

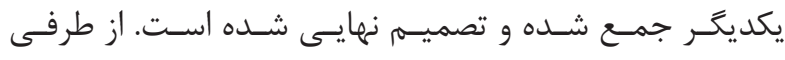

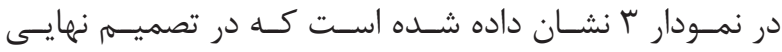

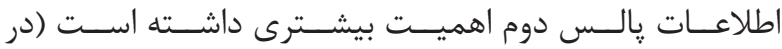

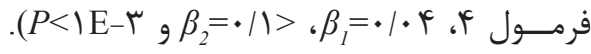

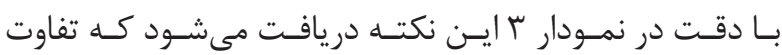

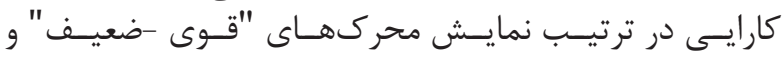

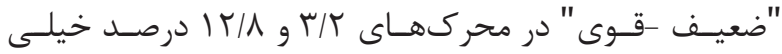

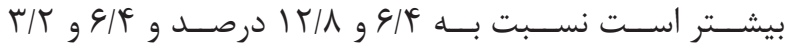

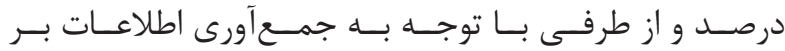

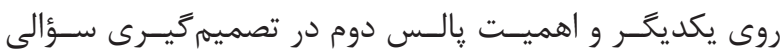

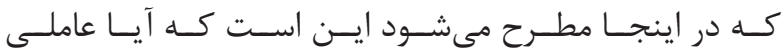

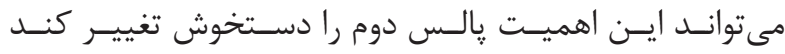

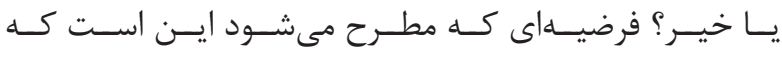

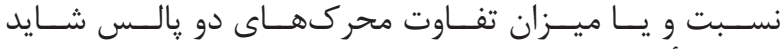

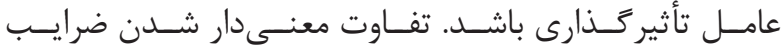
每

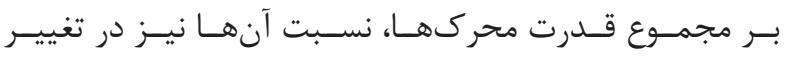

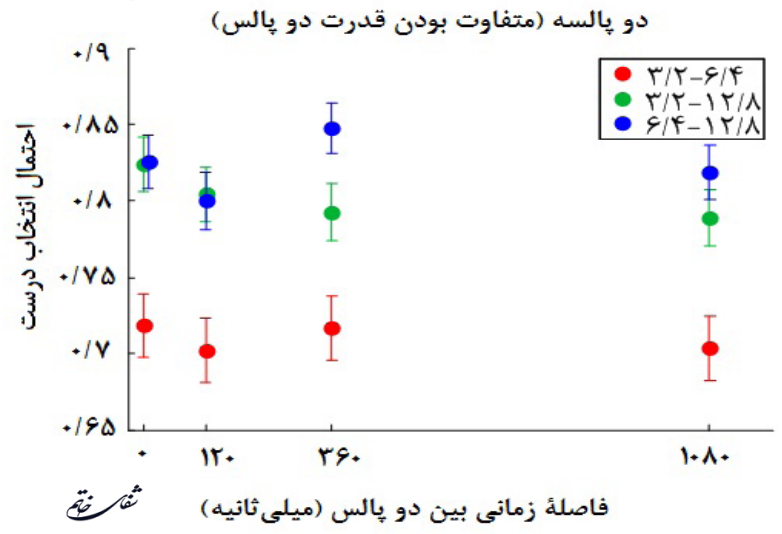

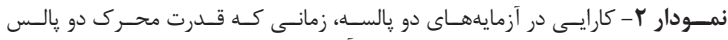

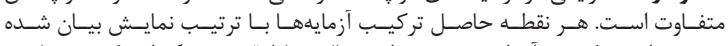

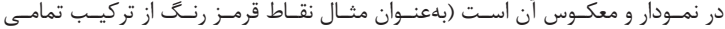

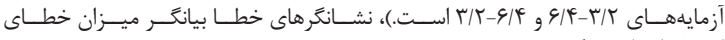

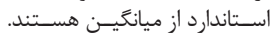

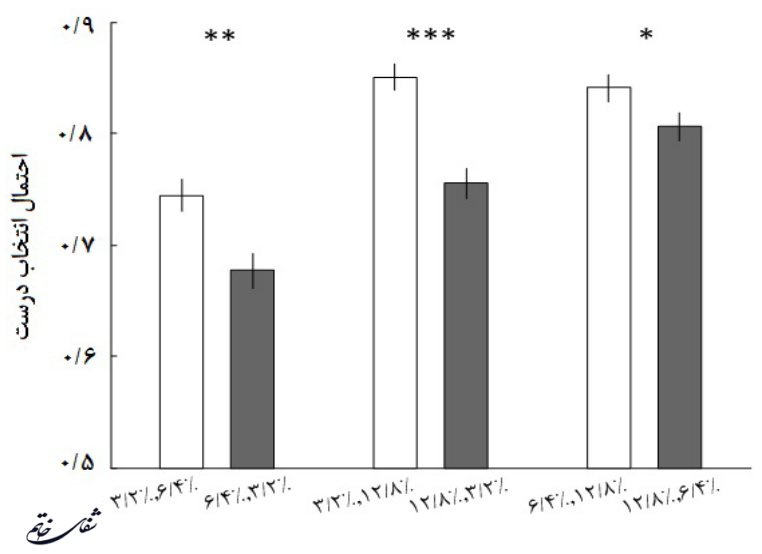

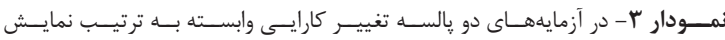

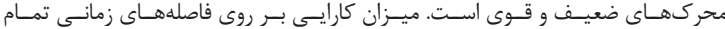

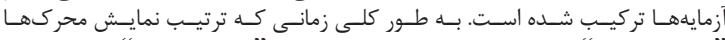

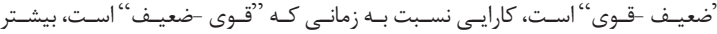

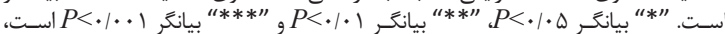

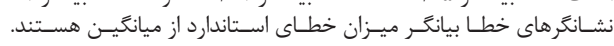




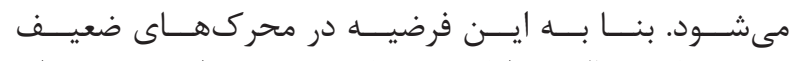

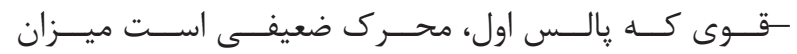

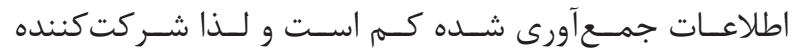

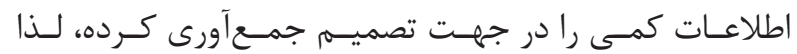

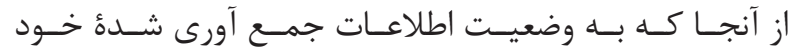

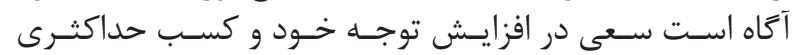

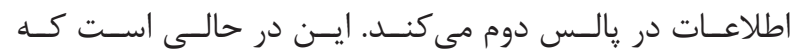

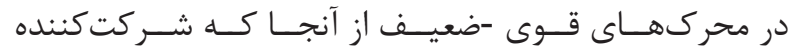

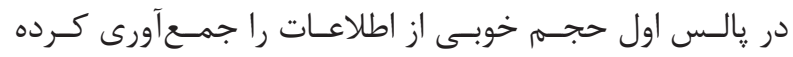

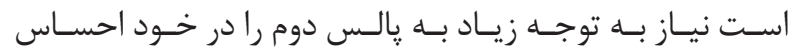

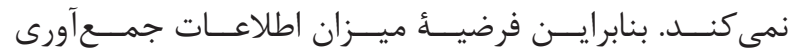

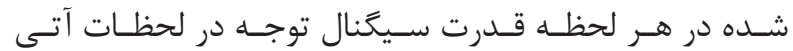

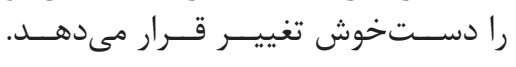

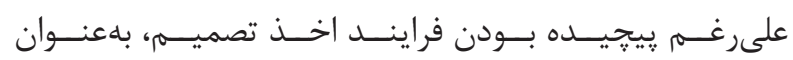

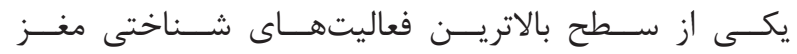

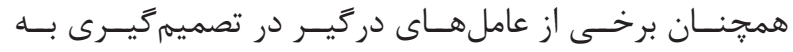

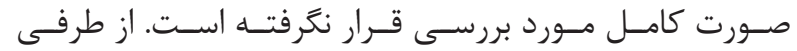

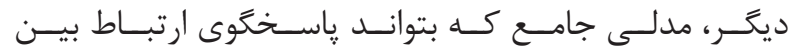

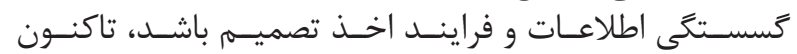

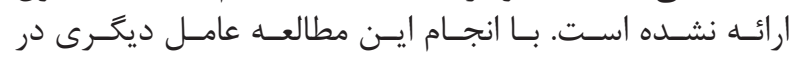

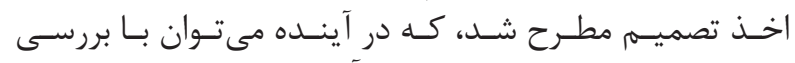

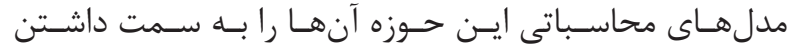

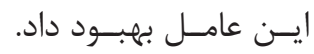

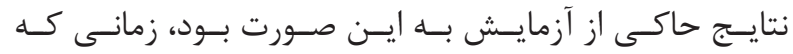

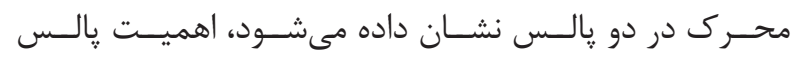

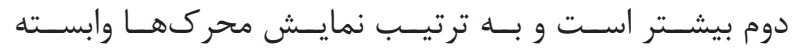

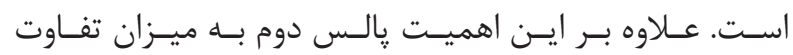

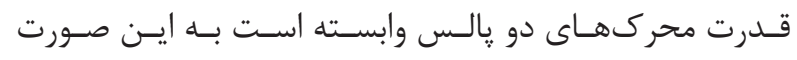

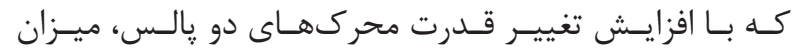

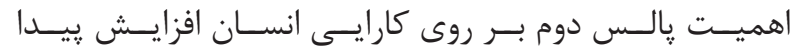

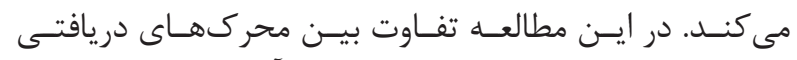

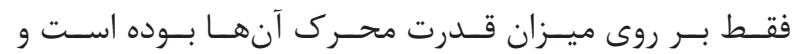

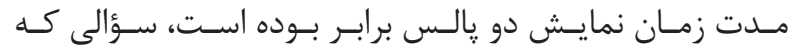

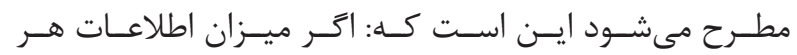

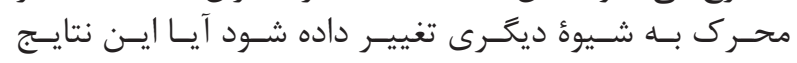

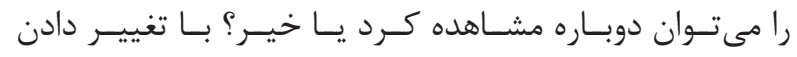

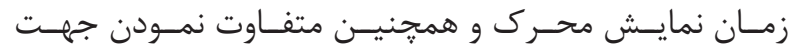

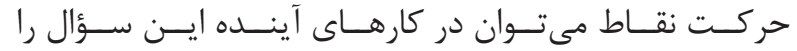

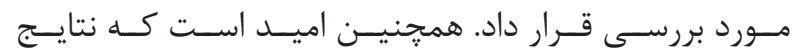

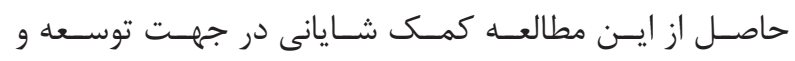

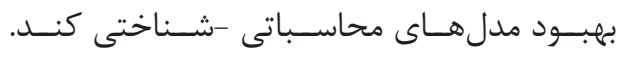

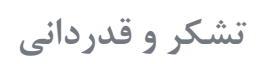

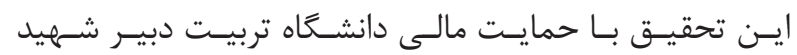

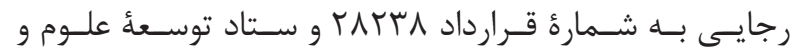

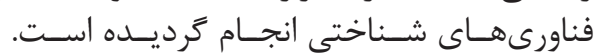

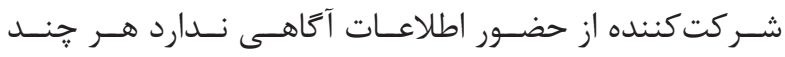

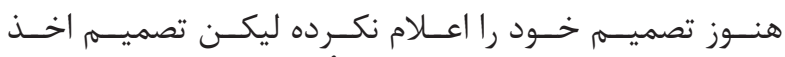

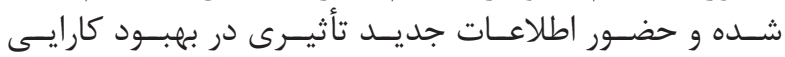

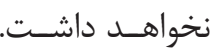

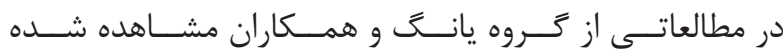

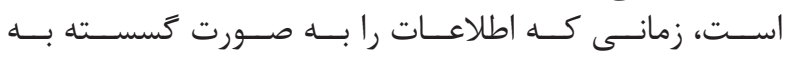

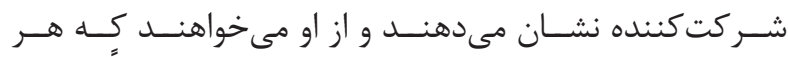

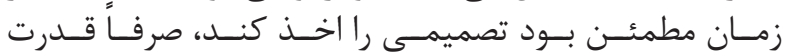

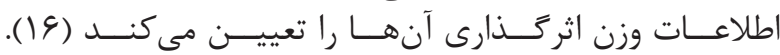

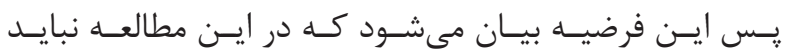

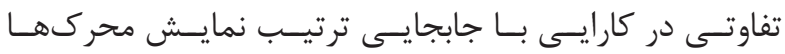

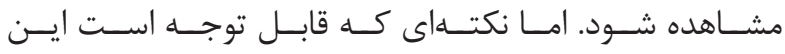

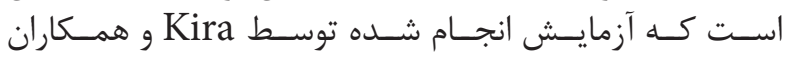

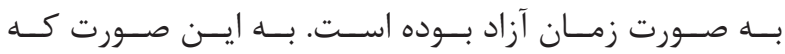

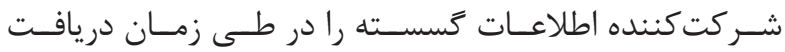

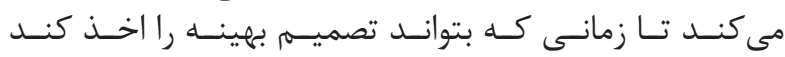

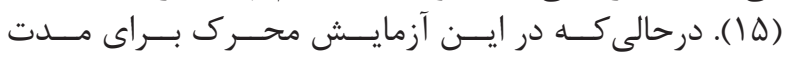

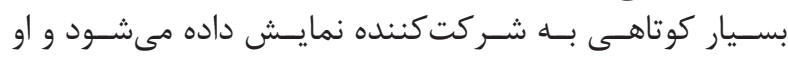

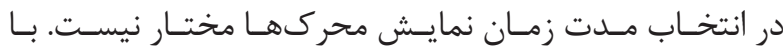

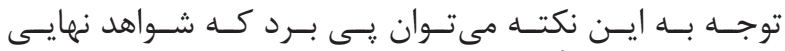

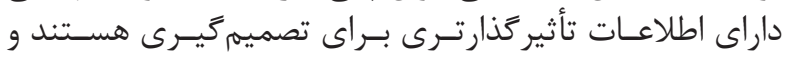

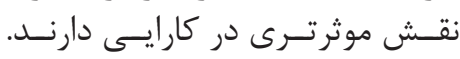

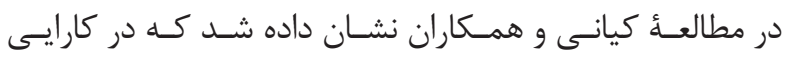

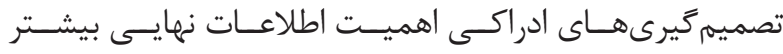

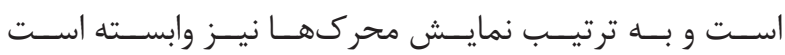

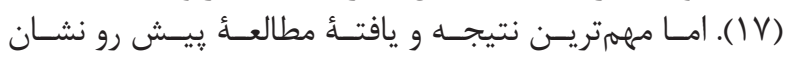

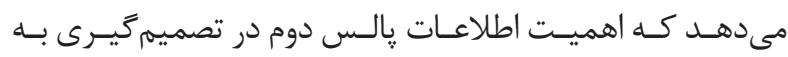

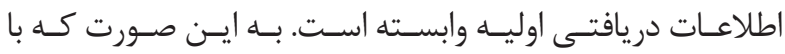

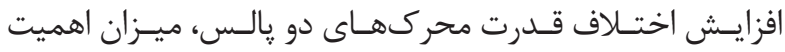

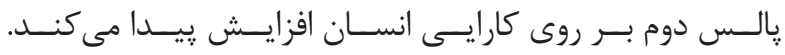

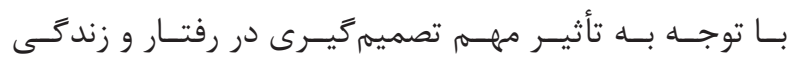

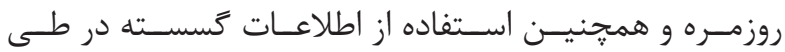

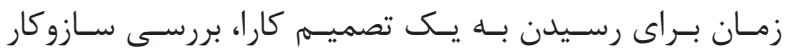

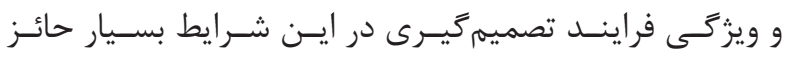

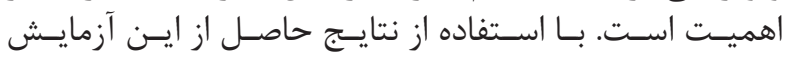

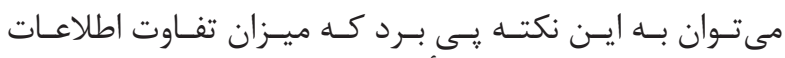

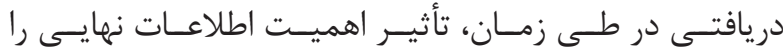

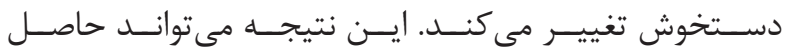

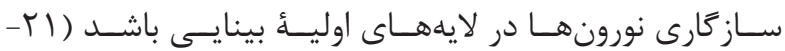

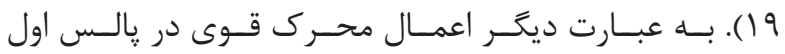

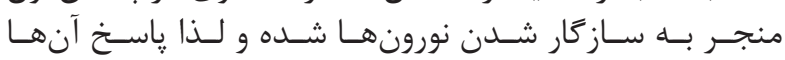

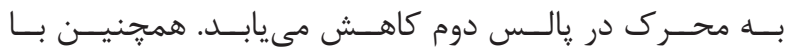

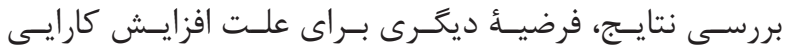

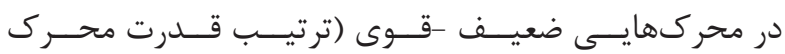

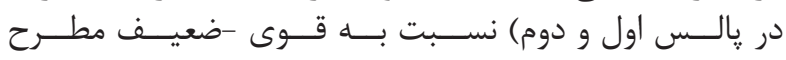


1. Gold JI, Shadlen MN. The neural basis of decision making. Annu Rev Neurosci. 2007; 30: 535-74.

2. Gardelle Vd, Summerfield C. Robust averaging during perceptual judgment. Proceedings of the National Academy of Sciences. 2011; 108(32): 13341-6.

3. Glimcher PW. Decisions, uncertainty, and the brain: the science of neuroeconomics. Cambridge: A Bradford Book; 2004.

4. Kiani R, Hanks TD, Shadlen MN. When is enough enough? Nat Neurosci. 2006; 9(7): 861-3.

5. Britten K, Newsome W, Shadlen M, Celebrini S, Movshon J. A relationship between behavioral choice and the visual responses of neurons in macaque MT. Vis Neurosci. 1996; 13(1): 87-100.

6.Newsome WT, Britten KH, Movshon JA. Neuronal correlates of a perceptual decision. Nature. 1989; 341(6237): 52-4.

7. Shadlen MN, Newsome WT. Neural basis of a perceptual decision in the parietal cortex (area LIP) of the rhesus monkey. J Neurophysiol. 2001; 86(4): 1916-36.

8. Roitman JD, Shadlen MN. Response of neurons in the lateral intraparietal area during a combined visual discrimination reaction time task. J Neurosci. 2002; 22(21): 9475-89.

9. Wang XJ. Probabilistic decision making by slow reverberation in cortical circuits. Neuron. 2002; 36(5): 955-68.

10. Lo C-C, Wang X-J. Cortico-basal ganglia circuit mechanism for a decision threshold in reaction time tasks. Nat Neurosci. 2007; 9(7): 956-63.

11. Hanks T, Kiani R, Shadlen MN. A neural mechanism of speed-accuracy tradeoff in macaque area LIP. Elife. 2014; 3: doi: 10.7554/eLife.02260.

\section{منابع}

12. Kiani R, Shadlen MN. Representation of confidence associated with a decision by neurons in the parietal cortex. Science. 2009; 324(5928): 759-64.

13. Churchland AK, Kiani R, Shadlen MN. Decisionmaking with multiple alternatives. Nat Neurosci. 2008; 11(6): 693-702.

14. Hanks TD, Mazurek ME, Kiani R, Hopp E, Shadlen $\mathrm{MN}$. Elapsed decision time affects the weighting of prior probability in a perceptual decision task. The Journal of Neuroscience. 2011; 31(17): 6339-52.

15. Kira S, Yang T, Shadlen MN. Aneural implementation of wald's sequential probability ratio test. Neuron. 2015; 85: 861-73.

16. Yang $T$, Shadlen MN. Probabilistic reasoning by neurons. Nature. 2007; 447: 1075-80.

17. Kiani R, Churchland AK, Shadlen MN. Integration of direction cues is invariant to the temporal gap between them. The Journal of Neuroscience. 2013; 33(42): 16483-9.

18. Kiani R, Hanks TD, Shadlen aMN. Bounded integration in parietal cortex underlies decisions even when viewing duration is dictated by the environment. The Journal of Neuroscience. 2008; 28(12): 3017-29.

19. Clifford CW, Webster MA, Stanley GB, Stocker AA, Kohn A, Sharpee TO, et al. Visual adaptation: neural, psychological and computational aspects. Vision Res. 2007; 47(25): 3125-31.

20. Webster MA. Visual Adaptation. Annu Rev Vis Sci. 2015; 1: 547-67.

21. Wang XJ, Liu Y, Sanchez-Vives MV, McCormick DA. Adaptation and temporal decorrelation by single neurons in the primary visual cortex. J Neurophysiol. 2003; 89(6): 3279-93. 